\title{
Periodontal profile and radiographic characterization of the jaws in a patient with autosomal dominant osteopetrosis
}

\author{
Davi da Silva Barbirato', Mariana Fampa Fogacci', Mariana Arruda², \\ Monique Oliveira Rodrigues 3,4 and Leonardo Vieira Neto ${ }^{2}$
}

'Wolfgang C. Pfeiffer Laboratory of Environmental Biogeochemistry, Federal University of Rondônia (UNIR), Rio de Janeiro, Brazil, 2Department of Internal Medicine and Endocrine Section, Medical School and Hospital Universitário Clementino Fraga Filho, Federal University of Rio de Janeiro, Rio de Janeiro, Brazil, ${ }^{3}$ Division of Graduate Periodontics, Department of Dental Clinic, Federal University of Rio de Janeiro, Rio de Janeiro, Brazil and 'Faculdades São José, Rio de Janeiro, Brazil

Correspondence should be addressed to D d S Barbirato Email

davibarbirato@gmail.com

\section{Summary}

Osteopetrosis (OP) comprehends a rare group of conditions, presenting on radiographs increased bone density, deriving from irregularities in osteoclast differentiation or function. In the autosomal dominant osteopetrosis (ADO), some patients stay asymptomatic for some time, or only develop mild symptoms. The dental surgeon is often the first to presuppose the disease during routine imaging examinations, referring the patient to a specialized medical group. Furthermore, osteomyelitis is one of the major OP complications, and should be refrained through frequent dental monitoring. Signals of cortical interruption, sclerotic sequestra or periosteal new bone formation, should be looked for in these patients. Their dental management is complex and procedures encompassing bone tissue, such as implant procedures, tissue regenerations, tooth extractions, maxillofacial surgeries and orthodontic treatments, when elected, should be avoided. This case report describes a case of ADO with a diagnosis of moderate generalized chronic periodontitis, not statistically related to plaque index. This is the first case to describe such a condition, in which the systemic component and the altered bone metabolism seem to be related to the loss of periodontal apparatus, independent of the biofilm. Concerning prevention, we can reinforce the need for frequent dental monitoring to avoid further interventions in those cases.

\section{Learning points:}

- This paper reports a case in which the systemic component and the altered bone metabolism seem to have been related to the loss of periodontal attachment apparatus, independent of the biofilm.

- The periodontal damage observed in the OP patient was not related to the dental plaque, which leads us to suggest that the cases of periodontitis in OP patients should be diagnosed as periodontitis as a manifestation of systemic diseases.

- The periodontitis prevention should be longed for in OP patients thus, we propose that doctors responsible for patients with OP refer them to a dental service as soon as possible and that dentists should be aware of the preventive dentistry value as well as the most appropriate dental management for those cases. 


\section{Background}

Osteopetrosis (OP) comprehends a group of conditions, clinically and genetically heterogeneous, presenting on radiographs increased bone density, deriving from irregularities in osteoclast differentiation or function (1). In all forms of OP, these cells show reduced ability to resorb bone and incapacity to form the ruffled border and dissolve the inorganic bone matrix. The lack of bone resorption in OP can explain the bone fragility seen in these patients (2).

Epidemiological studies refer these as a rare group of diseases, the incidence of which, worldwide, is still unclear $(1,3)$. A systemic search of OP in Denmark, stated the prevalence of 5.5/100000 inhabitants with autosomal dominant type of the disease (3).

Two major forms of OP can be differentiated (4), based on their type of inheritance: autosomal dominant OP (ADO, also known as Albers-Schonberg disease) and autosomal recessive $\mathrm{OP}$ (ARO). Their symptoms range from slight to fatal ones. The ADO usually affect adults, in a more benign form (2), whereas children can present the ARO, or malignant infantile osteopetrosis, right after birth, a more severe and deathful form when left untreated $(2,4)$.

The development of OP can occur in a severe form, although, principally in ADO some patients stay asymptomatic for some time, or only develop moderate symptoms (2). In such cases, often the dental surgeon will be the first to presuppose the disease during routine imaging exams. So the contribution of the dental professional with the suspicion of the OP diagnosis followed by the referral to a specialized medical group is primordial. Furthermore, it is known that osteomyelitis is one of the major OP complications (1), and therefore, it should be refrained through the intense, frequent and preventive dental monitoring.

This case report aims to contribute to the research of OP and its implications in the dental management of patients affected by the disease. We describe a case of ADO with a clinical and radiographic diagnosis of moderate generalized chronic periodontitis, not statistically related to plaque index. To the best of our knowledge, this is the first case in which such a condition is described, highlighting the interrelationship between the systemic involvement of the individual and the diagnosis of periodontitis.

\section{Case presentation}

A 44-year-old male presented to the Medical School of the Federal University of Rio de Janeiro reporting pain in his left leg for two months, with functional incapacity. He had already suffered six low-impact fractures, in the right tibia (two fractures on the same site at 16 years of age) in the left humerus, right radius and, most recently, two in
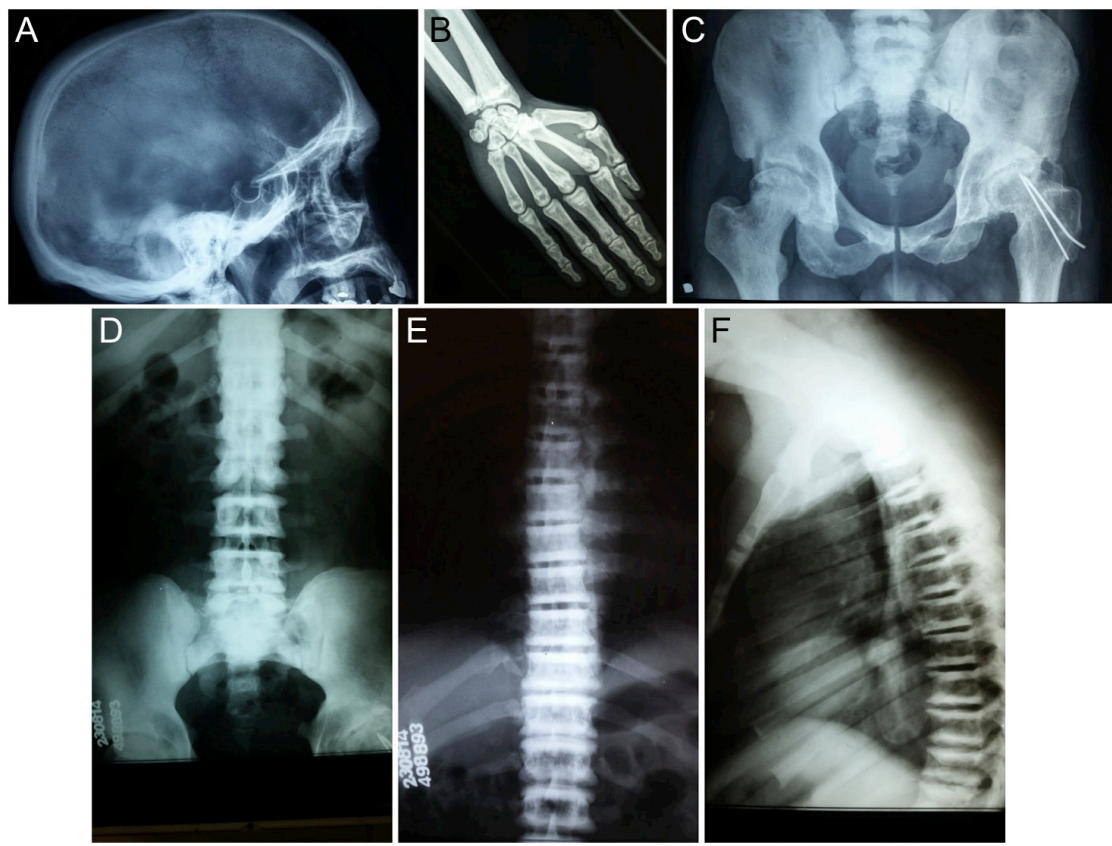

Figure 1

Bone alterations main findings. (A) Diffuse sclerosis of the skull base observed on X-ray. (B) Proximal phalanges with 'bone in bone' appearance. (C) Fracture of the femoral neck, narrow joint space, signs of prior fractures and thickening of the cortical layer. (D, E, F) Sclerosis of vertebral end plates (Rugger jersey spine). 
the left femoral neck. On physical examination, there was a shortening of approximately $1 \mathrm{~cm}$ of his left lower limb, a bilateral valgum thigh deformity and pain during mobilization of the leg. There was no visual loss or reduced hearing, and the height was consistent with his genetic target. Laboratory examination was all within the normal range, except for a high total acid phosphatase of $25.3 \mathrm{U} / \mathrm{L}$ (normal range $<6.6 \mathrm{U} / \mathrm{L}$ ). Hip X-ray showed a fracture of the femoral neck, narrow joint space, signs of prior fractures and thickening of the cortical layer. X-ray spine revealed the 'rugger jersey' pattern. Also, a diffuse sclerosis of the skull base could be noticed on X-ray (Fig. 1). The bone microarchitecture and the volumetric bone mineral density (BMD) were also assessed in this patient by a highresolution peripheral quantitative computed tomography (HR-pQCT), providing analogous measurements to those generated by a 2-dimensional (2D) histomorphometry and 3-dimensional microcomputed tomography of transiliac bone biopsies (5). He has already been submitted to seven surgical procedures because of fractures and joint deformities. Bisphosphonate drugs have never been prescribed to the patient. The patient reported a brother and a cousin with repetitive low-impact fractures, who were called for a clinical evaluation, unfortunately without success. His parents were nonconsanguineous. Data related to the diagnosis of OP for this patient have been recently published (5).

\section{Investigation}

The patient reported no smoking and no alcoholism. All the missing teeth have been surgically removed; there was no case of agenesis or retained tooth. The patient had

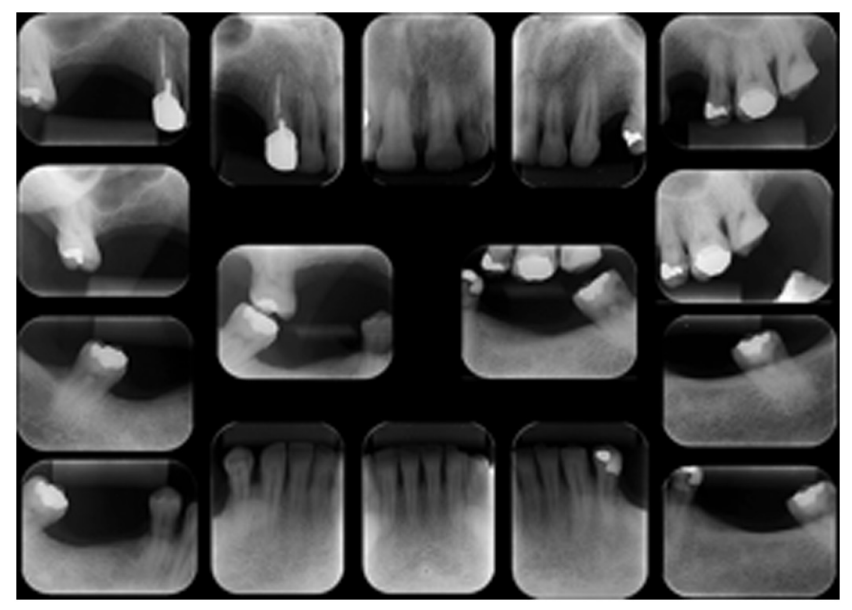

Figure 2

Periapical radiography complete exam and posterior bitewings. no buccal complaints and no signs or clinical symptoms compatible with odontogenic infection. In the periapical radiography complete exam, extensive restorations and carious lesions near the pulp chamber, and slight thickening of the apical periodontal space in the left first premolar tooth associated with an endodontic treatment and a root pin was observed. The radiography also revealed the absence of a radiopaque image related to the alveolar cortical bone, despite the no observed ankylosis (Fig. 2).

All permanent teeth, excluding third molars, received a complete periodontal examination at six sites per tooth. The parameters evaluated were plaque index (PI), calculus (CALC), gingival index (GI), bleeding on probing (BOP), probing depth (PD), clinical attachment loss (CAL), suppuration and tooth mobility. The PD and CAL were measured as the distance from the bottom of the periodontal sulcus/pocket to the free gingival margin and cementoenamel junction respectively. These measures were done in millimeters, rounded to the whole upper millimeter, and were presented as mean \pm standard deviation (s.D.). The PI, CALC, GI and BOP indexes (dichotomous variables) were analyzed as the relative frequency of periodontal sites affected.

The periodontal diagnosis was moderate generalized chronic periodontitis (6). Tooth plaque and calculus were detected in $59.6 \%$ and $25.8 \%$ of the periodontal sites respectively. The GI revealed blooding of the free gingival margin in $18.4 \%$, and the frequency of BOP $28.1 \%$. Mean $\mathrm{PD}$ and CAL were $2.77 \mathrm{~mm} \pm 0.82$ and $0.75 \mathrm{~mm} \pm 1.00$ respectively (Table 1 ). Tooth mobility and suppuration were not detected at any of the sites evaluated.

The PD and CAL variations were not related to the tooth plaque or the tooth calculus (Table 2). This result suggests that PI and CALC could not explain the periodontal pockets, or that they were slightly associated.

There was no statistical significance for the correlation between periodontal pockets ( $\mathrm{PD}>3 \mathrm{~mm}$ ) and PI, CALC and BOP. Also, there was no statistically significant correlation between CAL and PI, CALC and BOP (Table 3). These results lead us to suppose that the clinical signs of periodontal pocket activity (bleeding), the tooth plaque and the calculus could not explain the destructive periodontal disease observed in this case. It is possible that the systemic condition of this patient is an explanatory variable for this outcome. Also, the constant microbial and inflammatory injury directly in the alveolar bone could increase the risk of osteomyelitis in OP patients. 
Table 1 Description data of plaque index and calculus.

\begin{tabular}{lcc}
\hline Descriptive data & Values \\
\hline Probing depth & \\
Mean (s.D.) & $2.77(0.82)$ \\
Min; max & $1 ; 5$ \\
Severe periodontal pockets & $86.8 \%$ \\
Mild: $0-3 \mathrm{~mm}$ & $13.2 \%$ \\
Moderate: $4-5 \mathrm{~mm}$ & - \\
Severe: $\geq 6 \mathrm{~mm}$ & $0.75(1.00)$ \\
Clinical attachment loss & $0 ; 3$ \\
Mean (s.D.) & $34.2 \%$ \\
Min; max & $9.6 \%$ \\
Periodontitis & - \\
Mild: $1-2 \mathrm{~mm}$ & \\
Moderate: $3-4 \mathrm{~mm}$ & \\
Severe: $\geq 5 \mathrm{~mm}$ & \\
\hline
\end{tabular}

CAL, clinical attachment loss; Min., minimum; Max, maximum/PD 0-3 mm 4-5 $\mathrm{mm}$ and $\geq 6 \mathrm{~mm}$ - mild, moderate and severe periodontal pockets respectively (7)/CAL $1-2 \mathrm{~mm}, 3-4 \mathrm{~mm}$ and $\geq 5 \mathrm{~mm}$ - mild, moderate and severe periodontitis respectively (6); PD, probing depth.

\section{Treatment}

\section{Outcome and follow-up}

The patient is being folowed up at the Endocrinology and Orthopedic units. The patient presented with a new fracture in the tibia and will undergo orthopedic surgery. The oral health is being followed up at regular appointments.

\section{Discussion}

This paper reports a case of ADO pointing the dental health issue. In addition to the expected bone radiographic characteristics, a diagnosis of generalized moderate chronic periodontitis was set. A regression analysis indicated that the PI did not explain the periodontal damage, which leads us to suggest that the cases of periodontitis in OP patients should possibly be classified as periodontitis as a manifestation of systemic diseases (6) instead of chronic periodontitis.
The diagnosis of osteopetrosis was based on clinical data and mostly on the radiographic appearance of the skeleton (1). Our patient presented in his past history, six low-impact fractures, the first one being in right tibia (two fractures on the same site) at 16 years of age and posteriorly in left humerus, right radius and, most recently, two in left femoral neck. In addition, the main findings of bone alterations on this patient's X-ray include sclerosis of vertebral end plates (Rugger jersey spine), diffuse sclerosis of the skull base and proximal phalanges with 'bone in bone' appearance. It would be very interesting to have the molecular data of this patient so that we could try to relate the genetic mutation to phenotype; however molecular analysis was not performed.

Reporting to the scientific community a case of rare disease is always mandatory. Documenting that occurrence contributes to global epidemiological indices of the disease registered. Specifically for the dental community, publishing that record draws the attention of dental surgeons for the importance of a thorough radiographic analysis of patients. ADO sometimes being asymptomatic, often the dentist will be the first health professional to suspect the disease and refer the patient to a medical team.

Still, OP is a risk factor for osteomyelitis (8). Therefore, signals of cortical interruption, sclerotic sequestra in lowattenuation zones or periosteal new bone formation, should be observed in these patients, in regular dental appointments. We can also add the necessary caution in the dental management of these patients, both the preventive and the therapeutic aspects. About therapies, procedures encompassing bone tissue, such as implants procedures, tissue regenerations, tooth extractions, maxillofacial surgeries, orthodontic treatments, among others, when elected, should be avoided because of the risk of osteomyelitis.

A case of OP complicated by osteomyelitis of the maxilla and mandible has been reported (9). The authors

Table 2 Effect of the clinical parameters related to the etiology of periodontal diseases in the periodontal attachment level.

\begin{tabular}{|c|c|c|c|c|c|}
\hline \multirow[b]{2}{*}{ Dependent variable } & \multirow[b]{2}{*}{ Independent variable } & \multirow[b]{2}{*}{$\boldsymbol{R}^{2}$} & \multicolumn{2}{|c|}{ Confidence interval 95\% } & \multirow[b]{2}{*}{$P$ value } \\
\hline & & & Lower limit & Upper limit & \\
\hline \multicolumn{6}{|l|}{ Probing depth } \\
\hline & $\mathrm{Pl}$ & 0.006 & -0.109 & 0.510 & 0.201 \\
\hline & CALC & -0.008 & -0.478 & 0.360 & 0.781 \\
\hline \multicolumn{6}{|c|}{ Clinical attachment loss } \\
\hline & $\mathrm{Pl}$ & -0.008 & -0.431 & 0.336 & 0.807 \\
\hline & CALC & -0.002 & -0.289 & 0.740 & 0.386 \\
\hline
\end{tabular}

$\mathrm{CAL}$, clinical attachment loss; CALC, calculus/ $R^{2}$, adjusted determinating coefficient/linear regression analysis (significance level at $5 \%$ ); PD, probing depth; PI, plaque index. 
Table 3 Correlation (Pearson's test (two tailed)) between clinical parameters related to the etiology and activity of periodontal diseases and periodontal pockets.

\begin{tabular}{|c|c|c|c|}
\hline & PI & CALC & BOP \\
\hline \multicolumn{4}{|c|}{ Probing depth $>3 \mathrm{~mm}$} \\
\hline Correlation & 0.109 & -0.026 & 0.161 \\
\hline$P$ value & 0.250 & 0.782 & 0.087 \\
\hline \multicolumn{4}{|c|}{ Clinical attachment loss } \\
\hline Correlation & -0.023 & 0.082 & 0.056 \\
\hline$P$ value & 0.807 & 0.386 & 0.557 \\
\hline
\end{tabular}

BOP, bleeding on probing; CAL, clinical attachment loss; CALC, calculus; $\mathrm{PD}$, probing depth; $\mathrm{PI}$, plaque index.

stated a direct correlation between facial trauma and tooth extractions and the subsequent osteomyelitis incident, pointing the complexity of managing OP patients as the importance of preventive oral health care and dental/ periodontal assistance.

It is known that the much-talked bisphosphonateinduced osteonecrosis of the jaws has been compared to a chemically induced form of OP, with the clinical course of the disease similar to the genetically related form (10). Considering the adversities that often come from interventions in bisphosphonate users, and its complicated contention (10), it is proper to have much caution in the dental management of OP patients.

Concerning prevention and oral health promotion, we can reinforce the need for frequent and intense dental monitoring to avoid further interventions. Finally, the periodontitis prevention should be longed for in OP patients, since this paper reports a case in which the systemic component and the altered bone metabolism seem to have been related to the loss of periodontal attachment apparatus, independent of the biofilm.

In conclusion, this patient illustrates that periodontitis in OP patients might be classified as periodontitis as a manifestation of systemic diseases instead of chronic periodontitis. Thus, we propose that doctors responsible for the patients with OP should refer them to a dental service as soon as possible and that dentists should be aware of the preventive dentistry value as well as the most appropriate dental management for those cases.

\section{Declaration of interest}

The authors declare that there is no conflict of interest that could be perceived as prejudicing the impartiality of the research reported.

\section{Funding}

The authors are grateful to FAPERJ (Fundação Carlos Chagas Filho de Amparo à Pesquisa do Estado do Rio de Janeiro), CAPES (Coordenação de Aperfeiçoamento de Pessoal de Nível Superior) and CNPq (Conselho Nacional de Desenvolvimento Científico e Tecnológico) support.

\section{Patient consent}

The written informed consent has been obtained from the patient and the case report was submitted to the Ethical Committee of UFRJ Hospital Universitário Clementino Fraga Filho.

\section{Authors contribution statement}

All authors have viewed and agreed to the submission and have equally contributed to the case report. Drs Mariana Fogacci, Davi Barbirato and Monique Rodrigues were the periodontists responsible for the case and Drs Leonardo Vieira Neto and Mariana Arruda are the endocrinologists responsible.

\section{References}

1 Stark Z \& Savarirayan R 2009 Osteopetrosis. Orphanet Journal of Rare Diseases 4 5. (doi:10.1186/1750-1172-4-5)

2 Del Fattore A, Cappariello A \& Teti A 2008 Genetics, pathogenesis and complications of osteopetrosis. Bone 42 19-29. (doi:10.1016/j. bone.2007.08.029)

3 Bollerslev J 1987 Osteopetrosis. A genetic and epidemiological study. Clinical Genetics 31 86-90. (doi:10.1111/j.1399-0004.1987. tb02774.x)

4 Sobacchi C, Schulz A, Coxon FP, Villa A \& Helfrich MH 2013 Osteopetrosis: genetics, treatment and new insights into osteoclast function. Nature Reviews Endocrinology 9 522-536. (doi:10.1038/ nrendo.2013.137)

5 Arruda M, Coelho MC, Moraes AB, de Paula Paranhos-Neto F, Madeira M, Farias ML \& Vieira Neto L 2016 Bone mineral density and microarchitecture in patients with autosomal dominant osteopetrosis: a report of two cases. Journal of Bone Mineral Research 201631 657-662. (doi:10.1002/jbmr.2715)

6 Armitage GC 1999 Development of a classification system for periodontal diseases and conditions. Annals of Periodontology 4 1-6. (doi:10.1902/annals.1999.4.1.1)

7 Knowles JW, Burgett FG, Nissle RR, Shick RA, Morrison EC \& Ramfjord SP 1979 Results of periodontal treatment related to pocket depth and attachment level. Eight years. Journal of Periodontology $\mathbf{5 0}$ 225-233. (doi:10.1902/jop.1979.50.5.225)

8 Curé JK, Vattoth S \& Shah R 2012 Radiopaque jaw lesions: an approach to the differential diagnosis. Radiographics 32 1909-1925. (doi:10.1148/rg.327125003)

9 Trivellato AE, Ribeiro MC, Sverzut CE, Bonucci E, Nanci A \& de Oliveira PT 2009 Osteopetrosis complicated by osteomyelitis of the maxilla and mandible: light and electron microscopic findings. Head and Neck Pathology 3 320-326. (doi:10.1007/s12105-009-0142-1)

10 Marx RE, Sawatari Y, Fortin M \& Broumand V 2005 Bisphosphonateinduced exposed bone (osteonecrosis/osteopetrosis) of the jaws: risk factors, recognition, prevention, and treatment. Journal of Oral Maxillofacial Surgery 63 1567-1575. (doi:10.1016/j. joms.2005.07.010)

Received in final form 2 March 2017

Accepted 15 March 20172 March 201 